\title{
Photophoresis as a source of hot minerals in comets
}

\author{
O. Mousis ${ }^{1}$, J.-M. Petit ${ }^{1}$, G. Wurm ${ }^{2}$, O. Krauss ${ }^{2}$, Y. Alibert ${ }^{3}$, and J. Horner ${ }^{4}$ \\ 1 Institut UTINAM, CNRS-UMR 6213, Observatoire de Besançon, BP 1615, 25010 Besançon Cedex, France \\ e-mail: 0livier.Mousis@obs-besancon.fr \\ 2 Institute for Planetology, University of Münster, Wilhelm-Klemm-Str. 10, 48149 Münster, Germany \\ 3 Physikalisches Institut, University of Bern, Sidlerstrasse 5, 3012 Bern, Switzerland \\ 4 Astronomy Group, Open University, Walton Hall, Milton Keynes MK76AA, UK
}

Received 25 January 2007 / Accepted 24 February 2007

ABSTRACT

\begin{abstract}
A time-dependent model of the solar nebula is used to describe the outward transport of hot mineral aggregates from locations in the warm inner regions of the nebula under the influence of photophoresis. We show that there is a direct dependence between the size of the gap initially assumed to exist in the inner solar nebula and the heliocentric distance to which the aggregates are likely to drift. We demonstrate that, despite a significant contribution to the opacity of the disk resulting from Rayleigh scattering by hydrogen, photophoresis can be considered as a transport mechanism leading to the presence of hot minerals in comets. This mechanism can lead to an influx of hot minerals in the formation regions of the main cometary reservoirs, implying a potential "dust-loading" of bodies from these populations. This scenario is compatible with the detection of crystalline silicates in a growing number of comets and also with the recent identification of CAIs in the samples returned from Comet 81P/Wild 2 by the Stardust mission. Finally, we find that this mechanism is consistent with the compositional diversity observed in small bodies of the outer solar system, in contrast to other proposed processes which invoke an efficient turbulent mixing within the primordial nebula.
\end{abstract}

Key words. solar system: formation - comets: general - comets: individual: 81P/Wild 2, Kuiper Belt, Oort Cloud

\section{Introduction}

Crystalline silicates have been detected via remote sensing in a growing number of comets (Crovisier et al. 2000; Sitko et al. 2004; Wooden et al. 2004). Moreover, high-temperature minerals such as fosterite $\left(\mathrm{Mg}_{2} \mathrm{SiO}_{4}\right)$ and enstatite $\left(\mathrm{MgSiO}_{3}\right)$, along with grains resembling calcium-aluminium inclusions (CAIs), have been indentified in the samples returned from Comet 81P/Wild 2 by the Stardust mission (Brownlee et al. 2006, hereafter B06). All these minerals were formed at moderately to extremely high temperatures in the solar nebula (reaching 1400-1500 K for CAIs; Jones et al. 2000).

Since comets are presumed to have formed in the cold outer part of the solar nebula, several processes have been invoked in order to explain the origin of the detected high-temperature minerals. It has been suggested that shock waves in the outer solar nebula could anneal the amorphous silicates to crystallinity in situ prior to their incorporation in comets (Harker \& Desch 2002). However, there is no observational evidence for the existence of such processes. On the contrary, recent analysis of dust returned by the Stardust spacecraft from Comet 81P/Wild 2 reveals hot minerals which are incompatible with an origin by annealing of interstellar silicates in the primordial nebula (B06). Alternatively, the formation of crystalline silicates in the hot inner region of the solar nebula, and their rapid diffusive transport to the comet formation zone has also been proposed to explain the observations (Bockelée-Morvan et al. 2002). However, since the diffusive transport of crystalline silicates leads to a homogeneous distribution of grains within the solar nebula, this mechanism alone may not be consistent with the radial variations of the properties of minor planets, and large-scale differences between solar system bodies, that result from a non-efficient mixing within the disk (Gradie \& Tedesco 1982; Petit et al. 1999; B06).

In some thermodynamic formulations of the solar nebula (when the existence of an inner gap is postulated), the disk becomes optically thin enough for particles to see the proto-Sun, but still has a reasonable gas content, enabling the photophoretic force to push dust grains outward. This provides a mechanism to transport high temperature material from the inner solar system to the regions in which the comets were forming. Eventually, the dust driven outward in this manner will reach a region where the gas pressure is so low that the combined outward forces of radiation pressure and photophoresis can only balance the inward effects of gas drag. Depending on the nebula model considered, this can lead to a "pile-up" of dust between 5 and 23 AU. Such a process would result in an influx of hot minerals to the regions in which Edgeworth-Kuiper belt and Oort Cloud bodies were forming, which could result in a "dust-loading" of these bodies.

In this work, we use a time-dependent model of the solar nebula derived from Alibert et al. 2005 to describe the outward transport of particles in the form of hot mineral aggregates with sizes ranging between $10^{-5}$ and $10^{-1} \mathrm{~m}$ to the outer regions via photophoresis (Krauss \& Wurm 2005; Wurm \& Krauss 2006; Krauss et al. 2007). These aggregates are presumed to have been assembled from hot mineral individual grains ranging down to submicron sizes. We consider these hot minerals to have formed by condensation within the hottest portion of the solar nebula, well inside 1 AU (Chick \& Cassen 1997). From these assumptions, we show that, despite Rayleigh scattering adding a significant contribution to opacity in the nebula, photophoresis can be 
considered as an alternative transport mechanism to explain the presence of hot minerals in the formation regions of comets.

\section{Photophoresis in the solar nebula}

All particles embedded in gas and heated by light feel a photophoretic force, which usually pushes them away from the light source. The force is pressure dependent and can be stronger than radiation pressure and the Sun's gravity by orders of magnitude in the solar nebula. It requires that the solar nebula is sufficiently transparent, which is likely to become the case after several million years or less of evolution. As a result, particles ranging from micron to centimetre sized migrate in the late solar nebula under the combined action of photophoresis, radiation pressure and gas drag (Krauss \& Wurm 2005).

In a recent paper by Krauss et al. (2007, hereafter K07), we describe in detail how this drag can, in principle, clear protoplanetary disks at later times (transitional disks). Photophoresis has the ability to move matter from the inner to the outer system and back inwards again. For simplicity, in K07, it was assumed that the gaseous disk is transparent once the dust particles have moved out. However, a significant contribution to the opacity of a disk consisting solely of gas arises, at optical wavelenghts, from Rayleigh scattering by hydrogen (Mayer \& Duschl 2005). In this work, we investigate the influence of this opacity on the outward motion of particles engendered by photophoresis in the solar nebula.

For the sake of consistency, we use the same nebula model as K07. This model is calculated in the framework of the $\alpha$ formalism (Shakura \& Sunyaev 1973), and described in Alibert et al. (2005). It determines the midplane pressure, density and temperature, which are used to calculate the photophoretic force acting on dust particles. We assume that the gas surface density is initially given by a power law $\Sigma \propto r^{-3 / 2}$, with an initial value taken to be $\Sigma(5.2 \mathrm{AU})=600 \mathrm{~g} / \mathrm{cm}^{2}$. The mass of the nebula (between $0.25 \mathrm{AU}$ and $50 \mathrm{AU})$ is $\sim 0.05 M_{\odot}$. The photo-evaporation rate is taken as being equal to $\sim 1.5 \times 10^{-8} M_{\odot} / \mathrm{yr}$, leading to a nebula lifetime of the order of $\sim 4 \mathrm{Myr}$.

For temperatures below $1500 \mathrm{~K}$, the dominant dimming effect in the nebula at wavelengths shorter than a few $\mu \mathrm{m}$ is Rayleigh scattering from molecular hydrogen (Mayer \& Duschl 2005). This condition is fulfilled for the entire nebula after $10^{5} \mathrm{yr}$, with the temperature beyond $0.6 \mathrm{AU}$ falling below $1000 \mathrm{~K}$ already at this early stage. For $\mathrm{H}_{2}$, the Rayleigh scattering cross section is $\sigma(\lambda)=8.49 \times 10^{-45} / \lambda^{4}\left(\mathrm{~cm}^{2}\right)$ (Vardya 1962). Assuming the illuminating light follows a black body spectrum, one can compute the Planck mean cross section as a function of the black body temperature $T_{\mathrm{B}}, \sigma\left(T_{\mathrm{B}}\right)=1.54 \times 10^{-42} T_{\mathrm{B}}^{4}\left(\mathrm{~cm}^{2}\right)$ (Dalgarno \& Williams $1962^{1}$ ). Assuming a mean molar mass of $2.34 \mathrm{~g} / \mathrm{mol}$ for the disk, which corresponds to the value employed to describe its structure, this results in a mass absorption coefficient of $\sigma_{m}\left(T_{\mathrm{B}}\right)=3.96 \times 10^{-19} T_{\mathrm{B}}^{4}\left(\mathrm{~cm}^{2} / \mathrm{g}\right)$. Using the current effective temperature of the Sun $T_{\mathrm{B}}=5770 \mathrm{~K}$, we get $\sigma_{m}(5770)=4.4 \times 10^{-4}\left(\mathrm{~cm}^{2} / \mathrm{g}\right)$, while using $T_{\mathrm{B}}=6000 \mathrm{~K}$ for a brighter early Sun, we get $\sigma_{m}(6000)=5.1 \times 10^{-4}\left(\mathrm{~cm}^{2} / \mathrm{g}\right)$. For the rest of this work, we adopt a value of $\sigma_{m}=5 \times 10^{-4}\left(\mathrm{~cm}^{2} / \mathrm{g}\right)$. Light becomes extinguished close to the star as a result of the high gas density, while the outer regions play little role in the extinction.

To estimate the degree of particle transport to the cometary region while taking into account the scattering of light by the

1 In our case, $T_{\mathrm{B}}$ is not the temperature of the nebula, but rather the effective temperature of the illuminating source, the Sun.

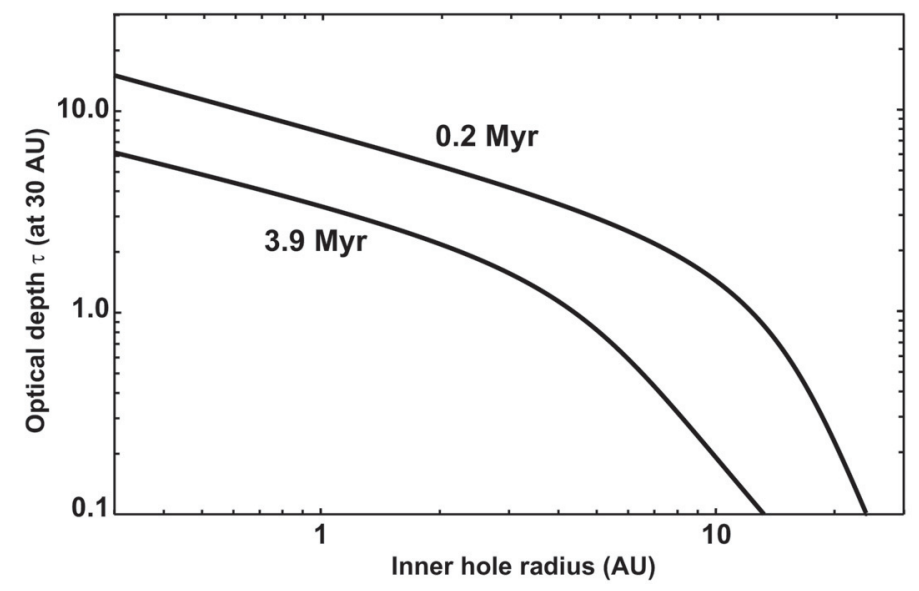

Fig. 1. Optical depth at $30 \mathrm{AU}$ as a function of the size of the inner hole in a disk dominated by Rayleigh scattering. The upper and lower curves are for $t=0.2$ and $3.9 \mathrm{Myr}$, respectively.

gas, we calculate the optical depth from an assumed inner edge of the disk to a distance of $30 \mathrm{AU}$. The results of this integration are seen in Fig. 1 for our disk model at $t=0.2 \mathrm{Myr}$ and $t=3.9$ Myr. Integrating along the mid-plane radial axis of the model from $0.25 \mathrm{AU}$ (the inner edge of the disk), the optical depth at $30 \mathrm{AU}$ (and further out) is initially about $\tau=17$. It decreases over time to $\tau=7$ at the end of the evolution of the disk. As a result, only $\sim 0.1 \%$ of the star's radiation will be available in the outer regions of the disk at late times. It should be noted that such high extinction resulting from Rayleigh scattering still allows the outward transport of solid particles to a few AU from the innermost regions. On the other hand, particle transport can be enhanced at larger heliocentric distances when a larger gap is formed in the inner disk. Indeed, there is a growing body of observational evidence for the existence of disks which have their inner few AU cleared or strongly depleted of gas (Sicilia-Aguilar et al. 2006). In the case of an inner hole of radius a few AU (Fig. 1), a reasonable value of $\tau$ (say $\tau=3$, which still leaves $5 \%$ of the incoming radiation reach the outer regions of the disk) is reached during the course of viscous evolution of the disk, and photophoresis essentially works as proposed by K07.

Now, we examine the migration of spherical hot mineral aggregates initially located in the inner solar nebula, where the opacity due to Rayleigh scattering dominates, and that were transported by photophoresis to the outer part of the disk. We have calculated the net motion of dust aggregates with sizes varying between $10^{-5} \mathrm{~m}$ and $10^{-1} \mathrm{~m}$, emissivity of 1 , thermal conductivity of $10^{-3} \mathrm{~W} \mathrm{~m}^{-1} \mathrm{~K}^{-1}$ and density of $\sim 500 \mathrm{~kg} / \mathrm{m}^{3}$ (a value corresponding to the random deposition of irregular olivine particles with density of $3300 \mathrm{~kg} / \mathrm{m}^{3}$, with a $15 \%$ filling factor; Blum \& Schräpler 2004). The influences of the photophoretic, radiation presure and residual gravity forces $\left(F_{\mathrm{ph}}\right.$, $F_{\text {rad }}$ and $F_{\text {res }}$ ) were taken into account in an evolving turbulent model of the solar nebula, using the formalism described in K07.

The radial drift velocity with respect to the nebula is then estimated to be

$v_{\mathrm{dr}}(a)=\frac{F_{\mathrm{ph}}(a)+F_{\mathrm{rad}}(a)+F_{\mathrm{res}}(a)}{m_{\mathrm{p}}} \tau_{\mathrm{g}}(a)$,

where $\tau_{\mathrm{g}}(a)$ is the gas grain coupling time, $m_{\mathrm{p}}$ the particle mass and $a$ is the radius of the particle (see K07 for details). Particles are pushed away via photophoresis while, at the same time, being dragged back towards the central star by the infalling nebula flow which moves at the velocity of $v_{\mathrm{ac}}$. In our disk model, the 


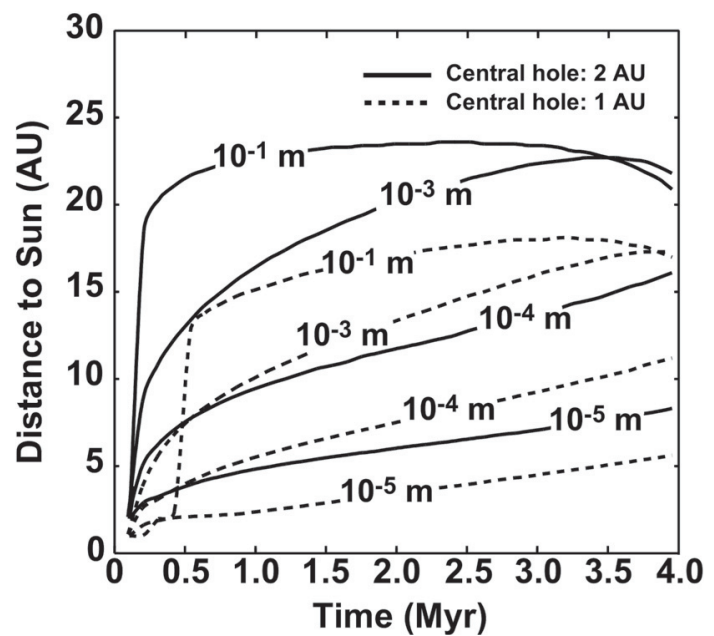

Fig. 2. From bottom to top: position of particles of size $10^{-5}$ to $10^{-1} \mathrm{~m}$, as a function of time and of the radius (AU) of the inner gap in the nebula. Positions of particles of size $10^{-2}$ to $10^{-1} \mathrm{~m}$ remain superposed with time.

accretion speed ranges from a few tens of $\mathrm{cm} / \mathrm{s}$ in the inner part to below one $\mathrm{cm} / \mathrm{s}$ at large distances in the early stages, and decreases substantially later on. In the simplified model presented here, we estimated the accretion velocity as

$v_{\mathrm{ac}}=\frac{R}{2 t_{\mathrm{vis}}}$

where $R$ is the distance from the Sun, $t_{\mathrm{vis}}=\frac{1}{3 \alpha} \frac{R^{2}}{H^{2}} \frac{1}{\Omega}$ is the typical local viscous time, $H$ is the local height of the nebula, $\Omega$ is the local Keplerian frequency and $\alpha$ is taken as being 0.002 . Finally, the position of particles is integrated from the inner edge of the disk at time $t=0$ to a position $R\left(t_{\text {disk }}\right)$ at the age of the disk $t_{\text {disk }}$

$R\left(t_{\text {disk }}\right)=\int_{0}^{t_{\text {disk }}}\left(v_{\text {dr }}(a, R(t), t)-v_{\text {ac }}(R(t), t)\right) \mathrm{d} t$.

Figure 2 shows the position reached by the aggregates as a function of time for an initial inner hole radius of 1 AU (dashed lines) and $2 \mathrm{AU}$ (solid lines). In both cases, the largest particles $\left(10^{-3}\right.$ to $\left.10^{-1} \mathrm{~m}\right)$ reach an equilibrium where the outward drift just balances the accretion flow, and hence rebound slightly toward the Sun during the late stages. Note that these particles move back inwards together, showing little differentiation during this inward motion. The smaller particles, however, evolve significantly slower, and continue their outward motion until the end of the calculations.

We also studied the case of a very small inner hole of 0.25 AU. In this case, no particle moved beyond 2 AU before $3 \mathrm{Myr}$, and only particles of size $10^{-3}$ to $10^{-2} \mathrm{~m}$ reach distances of $5 \mathrm{AU}$ or greater by the end of the integration.

\section{Implications for comets}

It is commonly acknowledged that comets were not formed in orbits similar to those in which they are now observed. Theories of the main source region for the Oort Cloud vary, from the suggestion that the bulk of objects therein formed near Jupiter and Saturn (Whipple 2000), and were ejected by these massive planets, to others where the vast majority of objects in the Oort Cloud formed further out, and were ejected primarily by Uranus and Neptune. The majority of the bodies within the EdgeworthKuiper Belt are presumed to have formed at a distance further from the Sun than that of Neptune (which, prior to migration, could have been located at a semi-major axis of between 15 and 20 AU) (Gladman 2005). Objects from the Edgeworth-Kuiper belt are also one possible source for the Jupiter Family comets (JFCs) (Horner et al. 2004), of which Comet 81P/Wild 2 is a member. The Scattered Disk contains a population of objects which lie beyond Neptune, but on significantly more eccentric orbits than the Edgeworth Kuiper belt objects. The Scattered Disk is expected to contain more cometary bodies which formed within the orbit of Neptune than the Edgeworth Kuiper belt and is expected to be the primary source of the JFCs (Duncan et al. 2004).

The mechanism proposed here implies that the heliocentric distance to which the dust can be transported is a function of the assumed size of the hole at the inner edge of the nebula. In the framework of our model, an inner gap of at least $1 \mathrm{AU}$ is required to allow high temperature dust to be pushed outward by photophoresis beyond the current orbit of Jupiter. If we assume that the smallest aggregates $\left(10^{-5} \mathrm{~m}\right)$ still create a prominent opacity in the disk, larger aggregates could only follow the small ones and reach $\sim 5.6 \mathrm{AU}$ at the end of the nebula's evolution (see Fig. 2).

By this time, it is clear that a significant fraction of the cometary bodies that formed in the vicinity of Jupiter would already have been ejected by the influence of the planet. For these bodies, under these circumstances, it is clear that photophoresis would be unlikely to play a significant role in the incorporation of hot material. Furthermore, many of the objects which eventually came to reside in the Edgeworth-Kuiper belt and Scattered Disk would be expected to have formed at distances significantly greater than 5.6 AU, and so, although their orbits would still be evolving at the end of the nebular lifetime, most would clearly reside at distances too great for photophoretic dust loading to play an important role. Though it is likely that some of today's comets remained in this region of the solar system for a longer period than the lifetime of the solar nebula (particularly in the case of the Main Belt Comets), one would expect objects from these areas to make up only a small minority of the active comets we observe. However, if the opacity engendered by the smallest particles was not significant in the disk (implying a dust size distribution dominated by large particles), then aggregates with sizes from $10^{-4}$ to $10^{-1} \mathrm{~m}$ should evolve outwards to further heliocentric distances. With such a size distribution, again assuming an inner gap of $1 \mathrm{AU}$, the position of aggregates of size $10^{-2}$ to $10^{-1} \mathrm{~m}$ reaches a distance of $10 \mathrm{AU}$ at $t \sim 0.5 \mathrm{Myr}$ in the nebula's life. At the end of the evolution of the disk, their position reaches $\sim 17$ AU. If we, instead, assume a somewhat larger inner gap of $2 \mathrm{AU}$, the $10^{-5} \mathrm{~m}$ grains move beyond a heliocentric distance of $5 \mathrm{AU}$ within $1.1 \mathrm{Myr}$, and reach $\sim 8.3 \mathrm{AU}$ at the end of the evolution of the disk. Aggregates of size $10^{-2}$ to $10^{-1} \mathrm{~m}$ are likely to drift towards greater heliocentric distances than the smaller ones, and settle at $\sim 20 \mathrm{AU}$ (or even further out) after $\sim 0.3 \mathrm{Myr}$. Interestingly enough, even particles of size $3 \times 10^{-4} \mathrm{~m}$, which correspond to the larger aggregates found in Comet 81P/Wild 2 (B06), also move beyond $20 \mathrm{AU}$, given these thermodynamic conditions within the nebula. Since it is postulated that comets accreted within a few hundred thousand years (Weidenschilling 1997), the dust transported via photophoresis can plausibly cross regions of the nebula in which cometary bodies have alrea dy formed. This would lead to many comets becoming "dust-loaded" by particles from the inner solar system, prior to their ejection by the giant planets, assuming the presence of an inner gap of at least 1 AU. The existence of crystalline silicates and CAIs reaching at least the micron size 
range that were found by the Stardust mission in the ejecta from Comet $81 \mathrm{P} /$ Wild 2 can be easily explained by our model. Indeed, the process we propose works if these particles are considered as building blocks of larger aggregates that drifted towards the outer solar nebula prior to being accreted by comets. Note that we talk about sizes of aggregates which can act as transport shuttles. As any number of small (even nanometre-size) grains could be part of a large aggregate, the size of such individual grains found in comets is eventually not related to the dynamically important size of the aggregates. Since photophoresis works heterogeneously, depending on the individual properties of a dust aggregate (composition, size, thermal and optical properties), one would expect the bulk of Oort-cloud comets to be laden with particles of different size and/or composition than those which fall upon Edgeworth-Kuiper Belt objects (or their daughter population, the JFCs).

It is important to mention that the mechanism we propose is in agreement with the compositional diversity observed in small bodies of the outer solar system. On the other hand, an efficient turbulent mixing scenario in the solar nebula, such as the one proposed by Bockelée-Morvan et al. (2002), would favor a homogeneous composition of these small bodies, in contrast with our current knowledge of their features.

Our model may be compatible with the observations of several comets by Sitko et al. (2004), where the authors concluded that Oort Cloud comets contain grains of a somewhat different nature to those seen in JFCs. However, these findings are contradicted by the more recent results derived from the Deep Impact mission to comet 9P/Tempel 1 (Harker et al. 2005; Sugita et al. 2005; Lisse, et al. 2006) which suggest that the dust seen in the ejecta of 9P/Tempel 1 seems similar to that observed in Oort Cloud comets, including Hale-Bopp. In fact, compositional differences have been inferred between the two JFCs 9P/Tempel 1 and $81 \mathrm{P} /$ Wild 2 but this may result from parent-body processing rather than compositional differences (B06).

Finally, we note that crystalline silicates supplied to preformed cometary bodies via photophoresis should be contained mainly in an outer dusty shell surrounding the cometary core. This might eventually be observable for comet fragments which are broken up after formation and dust mantling. In the case of the transport of such material by turbulent diffusion, the crystalline silicates should be homogeneously mixed with the other compounds within the cometary core.

Acknowledgements. G.W. and O.K. are supported by the Deutsche Forschungsgemeinschaft. Y.A. is supported by the Swiss National Science Foundation. J.H. gratefully acknowledges the support of PPARC. Many thanks to the anonymous Referee whose comments invited us to investigate in more details the range of possible parameters, thus strengthening our manuscript.

\section{References}

Alibert, Y., Mousis, O., \& Benz, W. 2005, ApJ, 622, L145

Beresnev, S., Chernyak, V., \& Fomyagin, G. 1993, Phys. Fluids A, 5, 2043 Blum, J., \& Schräpler, R. 2004, PhRvL, 93, 115503

Bockelée-Morvan, D., Gautier, D., Hersant, et al. 2002, A\&A, 384, 1107

Brownlee, D., Tsou, P., Aléon, J., et al. 2006, Science, 314, 1711

Chick, K. M., \& Cassen, P. 1997, ApJ, 477, 398

Crovisier, J., Brooke, T. Y., Leech, K., et al. 2000, Thermal Emission Spectroscopy and Analysis of Dust, Disks, and Regoliths, ASP Conf. Ser., 196, 109

Dalgarno, A., \& Williams, D. A. 1962, ApJ, 136, 690

Duncan, M., Levison, H., \& Dones, L. 2004, Comets II, 193

Gladman, B. 2005, Science, 307, 71

Gradie, J., \& Tedesco, E. 1982, Science, 216, 1405

Harker, D. E., \& Desch, S. J. 2002, ApJ, 565, L109

Harker, D. E., Woodward, C. E., \& Wooden, D. H. 2005, Science, 310, 278

Horner, J., Evans, N. W., \& Bailey, M. E. 2004, MNRAS, 354, 798

Jones, R. H., Lee, T., Connolly, H. C., Jr., Love, S. G., \& Shang, H. 2000, Protostars and Planets IV, 927

Krauss, O., \& Wurm, G. 2005, ApJ, 630, 1088

Krauss, O., Wurm, G., Mousis, O., et al. 2007, A\&A, 462, 977 (K07)

Lisse, C. M., VanCleve, J., Adams, A. C., et al. 2006, Science, 313, 635

Mayer, M., \& Duschl, W. J. 2005 MNRAS, 358, 614

Petit, J.-M., Morbidelli, A., \& Valsecchi, G. B. 1999, Icarus, 141, 367

Shakura, N. I., \& Sunyaev, R. A. 1973, A\&A, 24, 337

Sicilia-Aguilar, A., Hartmann, L., Calvet, N., et al. 2006, ApJ, 638, 897

Sitko, M. L., Lynch, D. K., Russell, R. W., et al. 2004, ApJ, 612, 576

Sugita, S., Ootsubo, T., Kadono, T., et al. 2005, Science, 310, 274

Weidenschilling, S. J. 1997, Icarus, 127, 290

Whipple, F. L. 2000, P\&SS, 48, 1011

Vardya, M. S. 1962, ApJ, 135, 303

Wurm, G., \& Krauss, O. 2006, Icarus, 180, 487

Wooden, D. H., Woodward, C. E., \& Harker, D. E. 2004, ApJ, 612, L77 